\title{
Transparencia de las entidades no lucrativas: criterios, metodología de evaluación y resultados
}

\section{Transparência de entidades sem fins lucrativos: critérios, metodologia de avaliação e resultados}

\section{Transparency of non-profit entities: criteria, evaluation methodology and results}

\author{
(iD) Pedro Molina Rodríguez-Navas \\ Universidad Autónoma de Barcelona, Barcelona, España \\ pedro.molina@uab.cat \\ (iD) Vanessa Rodríguez-Breijo \\ Universidad de La Laguna, Tenerife, España \\ vrbreijo@ull.edu.es
}

Resumen: En este trabajo, establecemos el marco conceptual de abordar la transparencia en las entidades no lucrativas y exponemos la metodología específica que hemos diseñado para evaluarla. Para ello, se ha contado con la participación de entidades y con sucesivas pruebas de validación. Asimismo, se ha desarrollado una plataforma web que ya están utilizando más de 200 organizaciones. Esta herramienta permite que entidades de diferentes tipologías jurídicas cuenten con indicadores y criterios ajustados a sus características. Finalmente, hemos analizado las webs de las entidades financiadas con más de 200.000€ anuales de subvenciones de carácter público y que, por tanto, tienen que cumplir lo establecido en la legislación de transparencia de Cataluña. Los resultados demuestran un índice de incumplimiento muy elevado. A la vista de estos resultados, consideramos imprescindible sensibilizar y formar a las entidades, ya que la Ley por sí sola no ha resuelto el problema.

Palabras clave: Transparencia. Entidades no lucrativas. ONG. Evaluación de la transparencia. Ley de Transparencia. 
Resumo: Neste trabalho, estabelecemos uma estrutura conceitual para abordar a transparência em entidades sem fins lucrativos e expor a metodologia específica que planejamos para avaliá-la. Para isso, contamos com a participação de entidades e com sucessivos testes de validação. Além disso, foi desenvolvida uma plataforma web que já está sendo usada por mais de 200 organizações. Essa ferramenta permite que entidades de diferentes tipologias jurídicas utilizem indicadores e critérios ajustados às suas características. Finalmente, analisamos os sites das entidades que recebem mais de 200 mil euros de subsídios públicos anuais e que, portanto, são obrigadas a cumprir a Lei de Transparência da Catalunha. Os resultados mostram uma taxa de não cumprimento muito alta. Em vista disso, consideramos essencial conscientizar e capacitar as entidades, uma vez que a Lei por si só não resolveu o problema. Palavras-chave: Transparência. Entidades sem fins lucrativos. ONG. Avaliação da transparência. Lei de Transparência.

Abstract: In this paper, we establish the conceptual framework to approach the transparency of non-profit entities and we describe the specific methodology we have designed to evaluate it. For this, it has counted on the participation of entities and with successive tests of validation. Likewise, we have developed a web platform that is already using more than 200 organizations. This tool allows entities of different legal typology to have indicators and criteria adjusted to their characteristics. Finally, we have reviewed the websites of entities receiving more than $€ 200,000$ of annual public aid and, therefore, are required to comply with the Catalan Transparency Law. The results show a very high default rate. In view of these results, we consider it essential to sensitize and train the entities, since the Law alone has not solved the problem.

Keywords: Transparency. Non-profit organizations. NGOs. Transparency evaluation. Transparency Law.

Data de recebimento do artigo: 30/01/2018

Data de aprovação do artigo: 24/07/2019 


\section{La importancia de la transparencia y el paradigma digital}

La transparencia es un valor y una actitud que la ciudadanía actual reclama en todos los aspectos que conforman la vida en sociedad. Se exige en la esfera gubernamental, pues se trata de sociedades democráticas, pero también como consumidores se solicita información sobre las compañías que producen bienes u ofrecen servicios. Desde el ámbito internacional hasta el local, surgen activistas de la transparencia y los creadores de programas de código abierto se manifiestan en contra de la propiedad de la información (SCHAUER, 2011).

Durantelaúltimadécada, tantolatransparencia comolarendición de cuentas se han posicionado como factores fundamentales en la lucha por el progreso económico y el correcto desarrollo de la democracia. Que los gobiernos e instituciones rindan cuentas es la mejor prevención contra la corrupción y la falta de eficiencia en la gestión. De esta manera el gasto público y las ayudas logran ser más eficaces. Ello incrementa las iniciativas sociales encaminadas al desarrollo, mejora los resultados y también su visibilidad (GAVENTA; MCGEE, 2013).

La transparencia, entendida como la apertura de las instituciones, implica, por un lado, el derecho de los ciudadanos a tener acceso a información sobre las actividades de las instituciones públicas y, por otro, la responsabilidad de estas instituciones de facilitar dicho acceso e información (KOSTADINOVA, 2015). En ese último aspecto se ubica la rendición de cuentas, a través de la cual las organizaciones explican el resultado de sus acciones, el impacto que han tenido en la mejora de la calidad de vida o el compromiso con la misión y los valores. Estos dos conceptos, transparencia y rendición de cuentas, están íntimamente relacionados, porque el acceso a la información es el primer paso en el proceso de rendición de cuentas. La transparencia condiciona el grado de información y la actitud con la que se realiza el proceso de rendición de cuentas (ROCHA VALENCIA; QUEIRUGA; GONZÁLEZ BENITO, 2015). 
Transparencia de las entidades no lucrativas: criterios, metodología...

No se trata solamente de tener las cuentas claras y comunicarlas, transparencia significa tener la suficiente apertura para revelar la estructura y el funcionamiento de una organización, dejar al descubierto cómo funciona por dentro, qué decisiones toma y cuáles son sus resultados. Pero esta determinación tiene que ser comprendida en toda su complejidad: no se trata nada más de justificar el dinero obtenido de los entes que la financian, sino de asumir la responsabilidad frente a los ciudadanosy a los beneficiarios, dejando claros los procesos que se van a ejecutar para lograr los objetivos propuestos (ARREDONDO TRAPERO; GARZA GARCÍA; VÁZQUEZ PARRA, 2014). Entendida de esta forma, solo existe transparencia si una organización provee la información suficiente sobre la totalidad de sus atributos, pero también si se comunica de manera oportuna y directa con cada una de sus audiencias más relevantes (LAPORTE; DEMCHAK; DEJONG, 2002).

En este sentido, los obstáculos que se generan por el espacio y el tiempo han podido ser superados gracias a las Tecnologías de la Información y la Comunicación (TIC), que hacen posible la circulación de contenidos e intercambios de forma instantánea, rápida e ilimitada (CRESPO RODRÍGUEZ; ZAFRA JIMENÉZ, 2005). Todo esto supone una gran oportunidad para la "transparencia electrónica", que se verifica cuando toda la información está real y efectivamente a disposición de los ciudadanos (GARCÍA COSTA, 2007).

Al respecto, Rosa Borge Bravo (2007) afirma que el uso de las TIC permite emitir y recibir información de primera mano, no filtrada por los medios de comunicación tradicionales, pero tampoco excesivamente mediatizada por las organizaciones. De esta manera, la ciudadanía puede ejercer cierto control sobre la labor de las personas que gestionan fondos públicos y se tiene la posibilidad de dar una respuesta eficaz e inmediata a la necesidad de información de distintos sectores. La internet permite que las personas puedan acceder a la información de las instituciones "de forma asequible, legible, comprensible, estructurada y reutilizable por cualquier persona física o jurídica" (CALVO, 2013, p. 72). De 
Transparencia de las entidades no lucrativas: criterios, metodología...

Pedro Molina Rodríguez-Navas • Vanessa Rodríguez-Breijo

esta forma, los ciudadanos se pueden formar una opinión, hacer valer sus derechos y pueden participar. Al mismo tiempo, se evita la corrupción y es un incentivo para aumentar la eficacia y competitividad (CALVO, 2013).

Gracias a las TIC, al menos potencialmente, es posible diseñar estrategias de participación del público en las decisiones que le afectan, como consultas en línea, foros, encuestas, peticiones, etc. El resultado debería ser una mayor implicación de los ciudadanos en el funcionamiento del sistema democrático (BORGE BRAVO, 2007). El uso de estas tecnologías facilita la difusión de información sobre cómo se está gestionando el dinero público, pero también crear un intercambio de ideas con las personas y con ello la construcción de una sociedad más transparente (BERTOT; JAEGER; GRIMES, 2010; Eom, 2014). A través de las páginas webs de las instituciones 0 corporaciones los ciudadanos pueden consultar en cualquier lugar y a cualquier hora a la información sobre cómo se está utilizando el dinero público, comunicarse entre sí y con los gestores de esos fondos y utilizar otros servicios en línea, con una inversión mínima para distribuir esos datos y ofrecer esas prestaciones (ROBERTS, 2006).

\section{La transparencia de las entidades sin ánimo de lucro: conocimiento, democracia y desarrollo}

Hace ya algo más de 100 años que Louis D. Brandeis (1914, p. 92) publicó su famosa cita: "Sunlight is said to be the best of disinfectants; electric light the most efficient policeman". Desde entonces, la transparencia ha seguido siendo tratada principalmente como un mecanismo preventivo de la corrupción. De hecho, en España la regulación sobre transparencia ${ }^{1}$ ha sido aprobada en un

1 Ley 19/2013, de 9 de diciembre, de transparencia, acceso a la información pública y buen gobierno. BOE, disponible en http://www. boe.es/boe/dias/2013/12/10/pdfs/BOE-A-2013-12887.pdf. 
Transparencia de las entidades no lucrativas: criterios, metodología...

clima de gran desconfianza política provocada por la revelación de múltiples casos de corrupción, en un contexto de crisis económica y empobrecimiento con graves consecuencias para sectores muy amplios de la ciudadanía.

Sinnegaresacualidad, sehanabierto paso otrasconsideraciones que impulsan la necesidad de aplicar políticas de transparencia por su repercusión en tres campos de cuestiones: conocimiento, democracia y desarrollo. Aunque las administraciones públicas sean las primeras instituciones obligadas a llevarla a la práctica y a impulsar cambios en esos ámbitos, tanto la legislación como la teoría contemplan que cualquier organización que reciba fondos públicos debe actuar de forma transparente y responder de sus actuaciones rindiendo cuentas (BLANES CLIMENT, 2014). Las entidades sin ánimo de lucro se encuentran entre estas de forma preferente, ya que su propia naturaleza las diferencia de otras organizaciones privadas por sus finalidades sociales y cívicas.

Es esencial que estas entidades privadas entiendan la transparencia como un valor fundamental, que deben incorporar considerando tanto la obligación legal que les exige cumplimiento como los beneficios que obtienen de aplicar nuevas prácticas socialmente reconocidas como positivas. Por el contrario, la opacidad es percibida por la ciudadanía como profundamente nociva e impropia de sujetos jurídicos, económicos y sociales que se proponen contribuir a mejorar diferentes aspectos del funcionamiento social (FERNÁNDEZ TORRES, 2013). El empoderamiento de los ciudadanos y la eficacia en la atención a las necesidades de las personas pasa necesariamente por una oportuna rendición de cuentas de quienes se suponen que están a su servicio (GAVENTA; MCGEE, 2013). Además, la transparencia es uno de los factores identificados claramente con la legitimidad de las organizaciones no gubernamentales (GUTTERMAN, 2013).

Sinembargo, hay diversosfactoresque retrasanlaincorporación de prácticas de transparencia por estas organizaciones. Algunos responden a falta de recursos materiales o humanos, otros a la falta de tradición, que implica escasa preparación y conocimientos 
Transparencia de las entidades no lucrativas: criterios, metodología...

insuficientes por parte de los técnicos, más acostumbrados a trabajar en el ámbito del marketing. Pero también hay resistencias porque la publicación de algunas de las informaciones se considera excesivamente sensible, al mostrar la estrategia de las entidades en un contexto también competitivo. Por tanto, se evita ofrecer información a las organizaciones del mismo sector, ya que se piensa que pueden utilizarla para tomar ventaja en el mercado, aunque este sea el de las subvenciones públicas. Estos factores pueden llegar a cuestionar la supervivencia de las organizaciones, como ya se apunta desde hace algunos años (HERRANZ DE LA CASA, 2007), puesto que socaban la credibilidad de las entidades en un entorno cada vez más exigente. "Responsabilidad" y "transparencia" corren el riesgo de ser palabras de moda, llenas de eufemismo y resonancia, pero vaciadas de su significado original (GAVENTA; MCGEE, 2013).

El peso de los problemas se está imponiendo a la evidencia de las aportaciones o beneficios que tienen para las entidades la incorporación del valor de la transparencia y la adopción de las prácticas que lo desarrollan, ventajas que, si se aplican, favorecen tanto a las entidades de forma individual como al conjunto del sector al conferirle un rasgo distintivo que las fortalece, que es socialmente valorado y demandado por la ciudadanía. Sin embargo, la aprobación de la legislación no ha difundido este cambio suficientemente y se está imponiendo una perspectiva legalista, de cumplimiento con las obligaciones, como si de un procedimiento administrativo más se tratara. Por el contrario, consideramos que la transparencia debe ser un valor inherente a las entidades sin ánimo de lucro y que su incorporación debe sincronizarse con procedimientos que garanticen su consolidación a la vez que han de mejorar la gestión de las entidades (FERNÁNDEZ; OSPINA, 2012).

Como hemos dicho, la transparencia es vista como necesaria en tres ámbitos esenciales de la vida social: el conocimiento, el fortalecimiento y profundización de la democracia, y el desarrollo económico. Estas tres esferas están claramente definidas en el ámbito de las instituciones públicas. Las organizaciones supraes- 
Transparencia de las entidades no lucrativas: criterios, metodología...

tatales han dado especial importancia a la relación entre economía y transparencia. Ya la Declaración del Milenio de la ONU relacionó transparencia y desarrollo económico al destacar, en el apartado III. Development and poverty eradication: "Success in meeting these objectives depends, inter alia, on good governance within each country. It also depends on good governance at the international level and on transparency in the financial, monetary and trading systems" (UNITED NATIONS, 2000, p. III, 13).

La Comisión Europea (2001) se propone mejorar, desde inicios de este siglo, su transparencia y la de las organizaciones internacionales en que intervenga, pero también señala que las organizaciones privadas activas a nivel europeo deben aplicar los mismos principios: "La propia sociedad civil debe aplicar los principios de buena gobernanza, entre los que se incluyen el de la responsabilidad y el de la transparencia" (COMISIÓN EUROPEA, 2001, p. 12).

En el caso de las entidades no lucrativas, es necesario ver cuáles son los contenidos a los que se refieren esos tres ámbitos. Aunque cada uno de ellos esté directamente relacionado con los otros dos, y resulte difícil segregar elementos, vamos a organizarlos en esos tres grupos para mostrárselos.

En cuanto al campo del conocimiento, es inherente a la transparencia la transmisión de información, pero estos dos conceptos no son equivalentes, ya que el conocimiento exige comprensión de la información y, por tanto, la transparencia requiere que los documentos complejos sean también explicados de forma inteligible para una audiencia no especializada. Por tanto, si bien la publicación de la información sobre el gobierno y la gestión de la entidad por sí sola mejora la confianza en la organización, es necesario que esta información sea comprensible para que ese efecto se cumpla completamente. Para los miembros de la organización, conocer lo que sucede en el conjunto de la entidad significa poder insertar su aportación en un contexto reconocible en el que adquiere un valor. Para las personas que acceden a la información sin formar parte de la entidad, conocer cualquier aspecto de la 
Transparencia de las entidades no lucrativas: criterios, metodología...

gestión la diferencia positivamente del resto de las organizaciones similares y crea un vínculo, un reconocimiento de esa organización como modelo positivo, lo cual incrementa las posibilidades de que la entidad sea valorada y que los sistemas de transmisión de información interpersonal y social tradicionales amplifiquen su repercusión. Herranz de La Casa (2007) refiere que la incorporación de la transparencia como valor mejora la imagen de la entidad, la confianza que proyecta y confiere reputación a largo plazo, incrementando así el valor de las organizaciones.

Por otra parte, la disponibilidad de la información permite que las organizaciones puedan contrastar sus procedimientos, servicios y soluciones, por lo que la extensión de las prácticas de transparencia permite que dispongan de modelos con los que mejorar su funcionamiento en todos los niveles. Porque "transparencia significa mostrar abiertamente el engranaje de una organización. Es decir, cómo funciona, cómo se lleva a cabo su trabajo, cuáles son los procesos internos, sus decisiones, los resultados obtenidos" (BAAMONDE-SILVA; PENA-RODRÍGUEZ; MARTíNEZ-ROLÁN, 2015, p. 29). Además y como consecuencia de lo anterior, la transparencia aporta nuevas oportunidades derivadas del conocimiento que obtienen tanto organizaciones similares como otras de muy diversa índole con las que pueden establecerse relaciones de colaboración y servicios.

Por último, continuando con esta dimensión, la transparencia propicia la presencia en los medios de comunicación, ya que el sistema mediático actual también se nutre de la información disponible en la red de Internet. Aparecer en los medios no es una cuestión menor para entidades que se proponen repercutir en la sociedad para posicionar nuevos valores o que buscan multiplicar su actividad e influencia. Además, la presencia en los medios puede suponer un incremento en los donativos, que finalizan al desaparecer estas campañas (FERNÁNDEZ TORRES, 2013). Las entidades sin ánimo de lucro pueden ser fuentes primarias de información, pero para ser consideradas como tales han de publicar las informaciones que las sitúan como valiosas. 
Transparencia de las entidades no lucrativas: criterios, metodología...

Pedro Molina Rodríguez-Navas • Vanessa Rodríguez-Breijo

El segundo ámbito es el de la mejora de la democracia de las entidades. En este sentido, la primera repercusión de la transparencia es la disposición de las informaciones que permiten el diálogo y la participación en las organizaciones en condiciones de igualdad. Sin disponer de la misma información es imposible el diálogo equitativo que permite una participación plena en los órganos de gobierno. Con transparencia se hace también posible el rendimiento de cuentas de esos órganos al conjunto de los miembros de la organización y se comunican los resultados de la actividad a través de las webs corporativas al conjunto de la sociedad, interesada en conocer cómo se distribuyen los recursos públicos y cómo y por quién son utilizados, para valorar si son justamente asignados. De hecho, en el ordenamiento jurídico la cuestión de la participación es fundamental, como lo demuestra la definición de transparencia de la Ley de Cataluña: ${ }^{2}$

Transparencia: la acción proactiva de la Administración de dar a conocer la información relativa a sus ámbitos de actuación y sus obligaciones, con carácter permanente y actualizado, de la forma que resulte más comprensible para las personas y mediante los instrumentos de difusión que les permitan un amplio y fácil acceso a los datos y faciliten su participación en los asuntos públicos (art. 2.a).

Por último, como hemos dicho, la transparencia tiene una influencia positiva en el ámbito económico. En primer lugar, porque previenela corrupción, tanto de prácticasque pueden serfraudulentas como de otras ilegales o alegales, enquistadas en las organizaciones y fuera del campo de visión de los observadores mientras no se han adoptado prácticas de transparencia. Recordemos que algunos de los casos de corrupción más conocidos de los últimos años en España han comprometido a organizaciones sin ánimo de lucro y que, por tanto, este no es un problema ajeno al sector.

2 Ley 19/2014, de 29 de diciembre, de transparencia, acceso a la información pública y buen gobierno, Comunidad Autónoma de Cataluña, BOE, núm. 18, de 21 de enero de 2015, páginas 4300 a 4342, disponible en https://www.boe.es/buscar/doc.php?id=BOE-A-2015-470. Consultado el 26/07/2017. 
Transparencia de las entidades no lucrativas: criterios, metodología...

Pero esta dimensión económica tiene su impacto más importante en relación también a la mejora de la percepción que tendrán otros agentes y personas que pueden aportar recursos económicos, como posibles donantes o inversores, recursos humanos, en el caso de los voluntarios o socios, o que pueden ofrecer nuevos servicios o posibilidades de colaboración contando con información sobre los proyectos y actividades que desarrolla una organización. Tampoco podemos olvidar que la legislación de transparencia obliga a todas las administraciones públicas a exigir el cumplimiento con las obligaciones de la Ley a las entidades que reciben financiación pública, siendo así un requisito imprescindible para optar a recursos económicos que para muchas entidades son fundamentales.

Para finalizar con este ámbito económico, hay que señalar que la incorporación de prácticas de transparencia tiene una repercusión directa en la mejora de la gestión, ya que obliga a reconsiderar tanto los flujos internos de información como las prácticas de la administración en su conjunto. La exigencia de publicar informaciones y documentos que anteriormente se consideraban internos obliga a plantearlos de nuevo, a estandarizarlos, así como a someterse a procesos más rigurosos de control de calidad y de calendarización de las actividades. Esto atañe a los diferentes ámbitos de la organización, incluyendo el técnico, financiero, administrativo, etc., y a las relaciones que se establecen entre ellos.

Además, consideramos imprescindible no olvidar un argumento fundamental: la transparencia es imprescindible para las organizaciones sin ánimo de lucro por coherencia con la misión y los valores de las organizaciones, que se proponen finalidades en términos de justicia social.

\section{Objetivos}

El primer objetivo de esta investigación ha sido diseñar una metodología de evaluación de la calidad y la transparencia de las 
Transparencia de las entidades no lucrativas: criterios, metodología...

Pedro Molina Rodríguez-Navas • Vanessa Rodríguez-Breijo

entidades no lucrativas, de utilidad para la investigación de carácter científico y para las propias entidades como procedimiento de autoevaluación y de mejora.

El segundo objetivo hasido aplicar esa metodología de evaluación a una muestra significativa, compuesta por entidades que tienen la obligación de observar lo prescrito en la Ley de Transparencia de Cataluña.

El avance del proyecto llevó a plantear un tercer objetivo: la conceptualización y el desarrollo de una plataforma web que pusiera a disposición de las entidades la metodología de autoevaluación y mejora, haciendo, así, una contribución en el proceso de implantación de las prácticas y de la cultura de la transparencia en ese sector. Además, el seguimiento de la utilización de esa herramienta ha de permitir ver cuál es la situación de estas organizaciones sobre la cuestión propuesta, sus dificultades y las necesidades.

\section{Metodología}

\section{Primera fase: revisión de antecedentes}

La metodología se ha fundamentado en los criterios y procedimientos desarrollados anteriormente en el proyecto Infoparticipa, que analiza la información publicada por las administraciones públicas locales en sus páginas web (MOLINA RODRÍGUEZ-NAVAS; SIMELIO SOLÀ; CORCOY RIUS, 2017). La metodología Infoparticipa utiliza una relación de indicadores de evaluación clasificados en bloques de información. Esa misma división ha servido para organizar los bloques temáticos de los indicadores que sirvieron para evaluar el nivel de transparencia de las entidades sin ánimo de lucro, pero adaptándolos y añadiendo uno inicial sobre la tipología y finalidades de la entidad, bloque que no era necesario en el caso de las administraciones públicas. Así, los bloques de información 
Transparencia de las entidades no lucrativas: criterios, metodología...

Pedro Molina Rodríguez-Navas • Vanessa Rodríguez-Breijo

de este proyecto son cinco: la entidad, los órganos y las personas que la gestionan y/o forman, cómo se gestiona, cómo se informa sobre ella y sus procedimientos de participación.

El Mapa Infoparticipa también ha sido el modelo para confeccionar las características de los indicadores de evaluación, formulados en forma de pregunta de respuesta binaria (sí o no consta la información en la página web) y la cuantificación de los resultados, en la que se consideran todos los indicadores con el mismo valor. Por último, la experiencia del proyecto Infoparticipa mostró la necesidad de contar con unos criterios de evaluación que aclarasen la formulación del indicador y los casos en que la validación podía ser positiva, por lo que los indicadores se completaron con criterios de aplicación y otras sugerencias sobre aspectos como la ubicación de las informaciones o sobre cómo mejorar la comprensión de las informaciones complejas.

Para definir los indicadores también se utilizaron otras referencias específicamente diseñadas para entidades sin ánimo de lucro, como el procedimiento del Observatori del Tercer Sector (VALLS, 2010), o el de la Fundación Lealtad, dirigido al sector de la cooperación ${ }^{3}$ y cuya finalidad es orientar y dar confianza a los donantes. También contrastamos la metodología de la Coordinadora ONG para el Desarrollo-España (COORDINADORA, 2012) y resultó especialmente interesante el trabajo de la Iniciativa Regional de la Sociedad Civil Rendir Cuentas (FERNÁNDEZ; OSPINA, 2012), desarrollado en el área latinoamericano.

En algunas ocasiones esas mismas metodologías han sido utilizadas en evaluaciones académicas, como la realizada por Gálvez, Caba y López (2009), quienes estudiaron 128 páginas web de ONGs que habían facilitado información a la Fundación Lealtad, por tanto entidades que en principio aplican prácticas de buen gobierno. Los resultados mostraron que la publicación de las informaciones en las webs no se correspondía con lo esperado, ya que el índice medio fue un $30,28 \%$. Los autores destacaron que en los aspectos relacionados con los fines y con la realización de

3 Ver metodología y resultados en Fundación Lealtad, disponible en: http://www.fundacionlealtad.org/web/home. 
Transparencia de las entidades no lucrativas: criterios, metodología...

proyectos los resultados fueron favorables, pero en los aspectos económicos más comprometidos, como la publicación de los presupuestos, los resultados fueron muy inferiores. Los mismos autores analizaron posteriormente (2012) una muestra de 55 organizaciones, en cuanto a las informaciones publicadas en las webs relacionadas con la responsabilidad social corporativa, resultando que las de mayor tamaño son las más transparentes. Por el contrario, los datos no mostraron una relación significativa entre transparencia y diversidad de género en la composición de los órganos de gobierno, ni entre transparencia y ámbito de actuación o antigüedad de las entidades.

También el Observatori del Tercer Sector aplicó su metodología (VALLS, 2010) a un análisis de 150 páginas web de organizaciones del tercer sector social. Los resultados fueron muy bajos, ya que solo el $64 \%$ de las entidades tenían web y eran muy pocas las que explicitaban su misión (18\%) o valores (16\%), y menos las que explicaban el origen de los recursos económicos (12\%). Además, el estudio señalaba la falta de claridad y una deficiente estructura de las páginas.

Todos estos trabajos se contrastaron con la legislación de transparencia de Cataluña, más exigente que la estatal, de forma que no solamente se ha utilizado el marco legal para la definición de indicadores y criterios. Este se ha enriquecido con las diferentes perspectivas y trabajos que han estudiado la misma cuestión, especialmente en los aspectos cualitativos pero también en algunos cuantitativos.

Segunda fase: participación de las entidades y construcción de la metodología

Con este bagaje construimos una primera relación de indicadores que, mediante una encuesta, se pidió que valoraran las personas de las entidades que asistieron a sesiones de formación sobre transparencia y calidad de la información. Los resultados de 
Transparencia de las entidades no lucrativas: criterios, metodología...

los 96 cuestionarios completados sirvieron tanto para definir mejor los indicadores como para comprobar la escasa cultura de la transparencia del sector, a pesar del compromiso verbal muchas veces manifestado, ya que los indicadores más exigentes recibieron un menor soporte. También permitió conocer mejor las expectativas y los deseos de las entidades, sus temores y resistencias, información muy útil para confeccionar el modelo definitivo.

Así, definimos una metodología de evaluación que puede ser utilizada también por las entidades como procedimiento de autoevaluación, que permite diagnosticar cuál es el estado de la transparencia de la organización, es decir, saber tanto con qué se está cumpliendo como con qué no. Se dispone así de un procedimiento de mejora que responde a tres propósitos:

a. Facilitar su comprensión para que tanto los técnicos especialistas como las personas socias o voluntarias de una organización o cualquier ciudadano pueda verificar una evaluación o aplicar una autoevaluación.

b. Incorporar elementos cuantitativos y cualitativos, de forma que evalúe la transparencia aplicando criterios de calidad de la información.

c. Conseguir que pueda ser utilizado tanto por las grandes organizaciones que deben cumplir con las legislaciones estatal y autonómica de Cataluña sobre Transparencia, como por las más pequeñas, que deben igualmente incorporar la transparencia como un valor inherente a la entidad.

En cuanto al primer propósito (a), se hizo un esfuerzo de redacción comprensible que fue completado por una adecuación para la edición final a los criterios internacionales de lectura fácil. Estos criterios contemplan tanto cuestiones relativas a la redacción como otros sobre la maquetación y el diseño que permiten incluso que las personas que presentan problemas físicos o cognitivos puedan acceder a los textos y comprender los contenidos. Para 
Transparencia de las entidades no lucrativas: criterios, metodología...

ello se encargó la revisión del trabajo a la Asociación Lectura Fácil, ${ }^{4}$ que representa en España a la organización internacional con base en Suecia.

Para incorporar tanto elementos cualitativos como cuantitativos (b), se acompañó cada indicador con una serie de criterios, considerando que transparencia y calidad de la información forman un binomio imprescindible. Sin calidad es imposible que la comunicación cumpla con objetivos tales como fomentar la participación. La cuestión entonces es determinar qué consideramos información de calidad. Para fijar sus características nos basamos en la Ley de Transparencia y en trabajos anteriores que abordan esta cuestión de forma específica. Finalmente, consideramos que la información de calidad ha de ser: completa y con datos primarios, pero a la vez inteligible al ser presentada en diferentes niveles de profundidad; publicada oportunamente, es decir, en el momento en que es de interés; fácilmente accesible y de forma no discriminatoria; presentada en formatos estandarizados, reutilizables y libres de licencia; y ha de responder a los criterios periodísticos de ser información humanizada, contextualizada y con memoria, además de ser contrastada y contrastable (MORENO SARDÀ et al., 2013).

Finalmente, la metodología no puede ser definida sin considerar la enorme diversidad de entidades existentes (c), atendiendo a su forma jurídica, al sector al que dedican su actividad o por su capacidad de trabajo, como resultado de los recursos de que disponen. Los indicadores y criterios se definieron contemplando estas diferencias, por lo que se diseñó el procedimiento dividiéndolo en cinco escalones. Los escalones 1 a 4 recogen la aplicación de la Ley, junto a los aspectos de lo que debe ser el estándar de calidad de transparencia, mientras que el quinto escalón contempla cuestiones específicas que no todas las organizaciones han de estar obligadas a incorporar y, por tanto, es de carácter voluntario y exclusivo para el procedimiento de autoevaluación y mejora. En cada escalón hay indicadores de todos o de algunos de los bloques de información y los criterios que se han seguido para dividirlos han

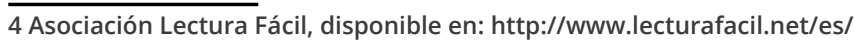


Transparencia de las entidades no lucrativas: criterios, metodología...

Pedro Molina Rodríguez-Navas • Vanessa Rodríguez-Breijo

sido la importancia de la información, la dificultad para elaborarla y la frecuencia necesaria de actualización.

Esta solución responde a diferentes motivos. En primer lugar, la división en escalones permite organizar los indicadores de forma coherente, mejorando la organización exclusivamente temática para conseguir que las informaciones fundamentales se incorporen antes que las más complejas. Así, por ejemplo, consideramos que los presupuestos o la planificación de una entidad no son posibles de comprender sin conocer previamente aspectos elementales como la misión de la entidad o su estructura organizativa, por lo que estas informaciones han de publicarse en primer lugar. Por otra parte, esta gradación consigue que las entidades puedan marcarse objetivos concretos, como puede ser alcanzar un determinado escalón del procedimiento, de forma realista y partiendo de sus necesidades y obligaciones. De esta forma, una gran entidad ha de plantearse llegar hasta el cuarto escalón, mientras que una entidad pequeña, con escasos recursos y actividad y que no ha de cumplir con la Ley, puede marcarse como meta llegar al escalón que le permita su capacidad.

Así, las entidades evalúan en qué escalón se encuentran y saben cuáles son los que les faltan, de forma que disponen de un procedimiento de mejora. Este método también permite que las entidades puedan decidir qué escalón aspiran alcanzar o hasta cuál deberían llegar en función de su capacidad.

\section{Tercera fase: pruebas y aplicaciones}

Construido el procedimiento, se publicó como guía, de forma abierta y en línea. ${ }^{5}$ Además de la repercusión que tuvo en las organizaciones, Francàs y Ginesta (2016) hicieron una investigación utilizando la metodología con 5 entidades de la comarca del Bergadà en Cataluña. Los autores evaluaron a las

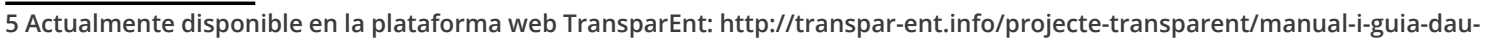
toavaluacio/. 
Transparencia de las entidades no lucrativas: criterios, metodología...

entidades considerando los 26 indicadores de los dos primeros escalones del procedimiento y los resultados de cumplimiento fueron: 17, 16, 13, 13 y 4 indicadores validados. Puesto que se trata de los dos escalones iniciales y que valoran las informaciones más elementales, los resultados son bajos.

Por nuestra parte, pusimos a prueba los indicadores y criterios asesorando a un grupo de entidades para que los pudieran aplicar. Este proceso se hizo a través de trabajo autónomo y de reuniones presenciales en las que se compartieron las dificultades y avances de cada una de ellas. La selección de las entidades se hizo considerando que debían participar aquellas que pudieran ser posteriormente referentes para otras entidades interesadas en aplicar el procedimiento y que debían estar representadas organizaciones de diferentes sectores y tipología. De las entidades que iniciaron el trabajo, 8 lo completaron y 2 no pudieron hacerlo por diferentes motivos.

\section{Revisión de la metodología y conceptualización de la plata- forma TransparEnt}

Este último trabajo mostró la necesidad de definir con más precisión algunos indicadores y de cambiar el orden de otros. Por otra parte, aunque los indicadores se definieron teniendo en cuenta que debía ser posible que cualquier tipología de entidad los pudiese aplicar, consideramos la necesidad de construir una herramienta que permitiera variaciones en función de la definición jurídica de las entidades. A la vez, la Generalitat de Catalunya (2015) publicó una guía para asociaciones y fundaciones que, aunque excesivamente ambigua, obligó a realizar algunos ajustes para mostrar la máxima coherencia en el campo de las obligaciones con la administración responsable.

Esta tarea llevó a concebir la plataforma web TransparEnt, de forma que las entidades pudieran acceder a una relación de indicadores mejor ajustados a su caso para hacer el procedimiento 
Transparencia de las entidades no lucrativas: criterios, metodología...

Pedro Molina Rodríguez-Navas • Vanessa Rodríguez-Breijo

de autoevaluación y mejora. Así, determinamos 4 (cuatro) grandes grupos de entidades: asociaciones y clubs deportivos, federaciones, fundaciones y entidades de nivel superior y otras clases de entidades (incluyendo entidades religiosas, cooperativas, etc.). Partiendo de un listado común de indicadores, hay algunas variaciones que se adecúan a los casos, por ejemplo en lo que respecta a la posibilidad de contar con personas asociadas o no, los órganos de gobierno (que difieren en función de la tipología jurídica), etc.

Presentamos, en la Tabla 1, la relación de indicadores(excluyendo los del escalón 5 por ser preguntas opcionales, o de respuesta voluntaria, que no forman parte del sistema de evaluación o autoevaluación), distribuidos en escalones y bloques de información, para la evaluación de las entidades de tipología "otros" tipos de entidades, ya que es la que presenta un redactado neutro que no alude a las circunstancias específicas de las otras tres categorías. Los criterios de aplicación de cada uno de estos indicadores definen con precisión las informaciones y condiciones de publicación necesarias para evaluar positivamente el indicador correspondiente y están disponibles en la plataforma para los usuarios registrados (no los exponemos en este trabajo por su extensión).

\section{Cuadro 1. Indicadores TransparEnt}

Escalón 1

Bloque 1. La entidad

1.1 ¿Se publican el nombre y los signos distintivos gráficos de la entidad?

1.2 ¿Se informa sobre la forma jurídica de la entidad?

1.3 ¿Se publica información sobre la misión, la visión y los valores de la entidad?

1.4 ¿Se publica información sobre cuáles son las personas o colectivos que pueden ser miembros, beneficiarios o usuarios?

1.5 ¿Se publica información sobre la creación e historia de la entidad?

1.6 ¿Se informa sobre las sedes de la entidad y la atención al público? 
Bloque 2. Órganos y personas que gestionan y/o forman la entidad

2.1 ¿Se publica información sobre cuáles son los órganos de gobierno?

2.2 ¿Se publica información sobre las funciones de los cargos que forman los órganos de gobierno?

2.3 ¿Se publica información sobre las personas que forman parte de los órganos de gobierno?

Bloque 3. Cómo se gestiona la entidad

3.1 ¿Se informa si la entidad es miembro o colabora con otras organizaciones públicas o privadas?

Bloque 4. Cómo se informa sobre la entidad

En este escalón no hay ningún indicador de este bloque.

Bloque 5. Procedimientos de participación

5.1 ¿Se informa del procedimiento a seguir para ser socio, voluntario o colaborador?

Escalón 2

Bloque 1. La entidad

1.7 ¿Se publican los estatutos?

1.8 ¿Se publica información sobre los aspectos más destacados de los estatutos?

Bloque 2. Órganos y personas que gestionan y/o forman la entidad

2.4 ¿Se publica un correo electrónico de contacto de cada una de las personas que forman parte de los órganos de gobierno?

2.5 ¿Se publica información sobre la trayectoria de las personas que forman parte de los órganos de gobierno?

2.6 ¿Se publica información estadística sobre socios, voluntarios y otras personas vinculadas a la entidad?

Bloque 3. Cómo se gestiona la entidad

3.2 ¿Se informa sobre la periodicidad con que se reúnen los órganos de gobierno?

3.3 ¿Se publica información sobre cuál es el marco legal que regula el funcionamiento de la entidad?

3.4 ¿Se publica información sobre otros documentos internos que regulan el funcionamiento de la entidad?

3.5 ¿Se publica el presupuesto anual?

3.6 ¿Se publica información sobre el origen de los ingresos?

3.7 ¿Se da información sobre qué parte del presupuesto se dedica a la ejecución de proyectos y actividades relacionadas con la misión de la entidad?

Bloque 4. Cómo se informa sobre la entidad

4.1 ¿Se publican noticias sobre la entidad y sobre las decisiones tomadas en las asambleas y demás órganos de gobierno?

4.2 ¿Se publica el nombre de la persona encargada de la comunicación de la organización y su contacto? 
Bloque 5. Procedimientos de participación

5.2 ¿Se informa de las actividades que pueden desarrollar los socios o voluntarios y de cómo pueden participar?

Escalón 3

Bloque 1. La entidad

En este escalón no hay ningún indicador de este bloque.

Bloque 2. Órganos y personas que gestionan y/o forman la entidad

2.7 ¿Se informa de si las personas que forman parte de los órganos de gobierno reciben retribuciones por trabajos prestados a la entidad?

2.8 ¿Se publica información sobre el organigrama ejecutivo y/o técnico?

2.9 ¿Se informa sobre la existencia de un consejo asesor o de otras personas asesoras, quiénes son y si son retribuidas?

Bloque 3. Cómo se gestiona la entidad

3.8 ¿Se publica información sobre la planificación del año en curso?

3.9 ¿Se publican las cuentas anuales del último ejercicio finalizado y las auditorías, en su caso?

3.10 ¿Se informa sobre el cumplimiento de las obligaciones tributarias y con la Seguridad Social?

3. 11 ¿Se publican las convocatorias y el orden del día de las asambleas con suficiente antelación?

Bloque 4. Cómo se informa sobre la entidad

4.3 ¿Se hacen en la web campañas de publicidad y/o se publican los materiales utilizados en otros soportes para difundir las actividades o captar socios, voluntarios, etc.?

4.4 ¿Se publica una agenda de actividades?

Bloque 5. Procedimientos de participación

5.3 ¿Se informa sobre la existencia de espacios de participación como grupos de trabajo, comisiones u otros?

5.4 ¿Se publican los enlaces a las redes sociales de la entidad?

Escalón 4

Bloque 1. La entidad

En este escalón no hay ningún indicador de este bloque.

Bloque 2. Órganos y personas que gestionan y/o forman la entidad

2.10 ¿Se publica información sobre el equipo de dirección/gestión y el equipo técnico?

Bloque 3. Cómo se gestiona la entidad

3.12 ¿Se publica un plan estratégico?

3.13 ¿Se publica información de seguimiento de los proyectos vigentes?

3.14 ¿Se publica información que permite valorar la calidad de los servicios y el grado de cumplimiento de los objetivos generales de la entidad y de sus proyectos? 
Transparencia de las entidades no lucrativas: criterios, metodología...

Pedro Molina Rodríguez-Navas • Vanessa Rodríguez-Breijo

3.15 ¿Se publican informes de seguimiento del presupuesto anual?

3.16 ¿Se publican las retribuciones de los máximos responsables de la gestión de la entidad y el convenio colectivo que regula las condiciones de trabajo del personal contratado?

Bloque 4. Cómo se informa sobre la entidad

4.5 ¿Se informa sobre la existencia de una política de transparencia?

Bloque 5. Procedimientos de participación

5.5 ¿Se informa de las condiciones en que se ejerce el voluntariado?

5.6 ¿Hay un buzón virtual de sugerencias y quejas?

Fuente: Elaboración propia.

\section{Plataforma web TransparEnt: funciones y utilización}

La plataforma web TransparEnt (disponible en www.transpar-ent. info) ofrece a las entidades cuatro funciones: hacer autoevaluación de su transparencia, seguir un procedimiento para mejorarla a partir del diagnóstico realizado, obtener documentos de autoacreditación del nivel que han podido alcanzar y disponer de ejemplos y referencias de buenas prácticas.

La plataforma se presentó el día 7 de abril de 2016 y hasta el 31 de diciembre de 2016 se habían registrado como usuarias 213 entidades. Siguiendo las tipologías de registro que diferencian la plataforma, 109 son asociaciones o clubs deportivos, 58 fundaciones, 26 federaciones $u$ organizaciones de nivel superior y 20 se han registrado con la categoría "otras".

Por sectores de actividad, una clara mayoría, 128 de 213, son entidades de acción social. El resto, en orden descendente, son culturales (19), dedicadas a la formación o educación (17), deportivas (10), comunitarias o vecinales (9), de cooperación o derechos civiles (8), juventud (4), medio ambiente (3) y 15 entidades más perteneciente a otros sectores de actividad. Esta clara mayoría de las entidades del tercer sector se debe a diferentes factores, como la especial sensibilidad en el campo de los valores de las 
Transparencia de las entidades no lucrativas: criterios, metodología...

Pedro Molina Rodríguez-Navas • Vanessa Rodríguez-Breijo

entidades dedicadas a la acción social o una mayor disposición de recursos para contar con profesionales.

Del conjunto de entidades registradas, 82 tienen la sede en la ciudad de Barcelona y el resto, 131, en otros lugares de Cataluña. Esta distribución territorial tiene su correlación en el ámbito territorial de actuación, ya que las no ubicadas en la ciudad de Barcelona tienen generalmente un alcance local o comarcal. El resultado final es que 20 tienen una dimensión internacional, 11 estatal, 76 autonómica, 68 comarcal y 38 local o vecinal.

Otro dato interesante es el presupuesto del último año que manifiestan las entidades y que se recoge en la Tabla 2. Como se puede ver, hay un claro predominio de las que tienen presupuestos por encima de 100.000 y de 500.000€, por tanto, las que cuentan con más recursos y las que han de cumplir con la Ley de Transparencia.

Tabla 2. Presupuesto del último año que declaran las entidades registradas en la plataforma TransparEnt (31 de diciembre de 2016)

\begin{tabular}{lc}
\hline & Número de entidades \\
\hline Hasta $5.000 €$ & 16 \\
\hline Entre 5.001 y $15.000 €$ & 14 \\
\hline Entre 15.001 y $50.000 €$ & 22 \\
\hline Entre 50.001 y $100.000 €$ & 24 \\
\hline Entre 100.001 y $500.000 €$ & 68 \\
\hline Más de $500.000 €$ & 69 \\
\hline
\end{tabular}

Fuente: elaboración propia.

A pesar de estos datos de utilización, hasta diciembre de 2016, de las 213 entidades registradas, solo 27 habían superado el primer escalón del procedimiento, 12 el segundo, 8 el tercero y 7 el cuarto. 


\section{Resultados de la evaluación de entidades perceptoras de subvenciones del Ayuntamiento de Barcelona}

Utilizandola metodologíaTransparEnt, en el primer trimestre de 2017 hemos hecho una evaluación de páginas web de las entidades que se han beneficiado de las subvenciones del Ayuntamiento de Barcelona. Para seleccionar la muestra, descargamos de la página web de la misma institución los datos relativos a las ayudas y subvenciones recibidas en el primer semestre de 2016. Estos datos los cruzamos con las informaciones sobre subvenciones y ayudas otorgadas por la Generalitat de Catalunya en 2016 en tres organismos diferentes y directamente relacionados con las actividades de las entidades sin ánimo de lucro: la Secretaría General de Deporte, la Dirección General de Cultura Popular y la Dirección General de Acción Cívica y Comunitaria.

Obtuvimos, así, una relación de entidades que habían recibido, en 2016, subvenciones y/o ayudas públicas que sumadas superaban $200.000 €$. Algunas solo han recibido estas cantidades económicas del Ayuntamiento de Barcelona y otras las han alcanzado sumando las de esta institución con las percibidas de los organismos de la Generalitat de Cataluña. Todas ellas han de cumplir inexcusablemente con ambas legislaciones de Transparencia, la nacional y la autonómica de Cataluña, ya que recibieron más de $100.000 €$ de ayudas o subvenciones públicas en una anualidad.

Utilizamos como muestra las 55 que habían recibido las cantidades más altas, entre 216.053 y 3.305.617€. De estas entidades evaluadas, 26 son fundaciones, 13 asociaciones, 10 federaciones, 3 cooperativas, 2 entidades religiosas y 1 consejo deportivo escolar.

El análisis arrojó estos resultados:

- 5 entidades no tienen ni tan solo página web. Para verificar este sorprendente dato, contactamos telefónicamente con cada una de ellas. 
Transparencia de las entidades no lucrativas: criterios, metodología...

- Excluyendo a las 5 entidades que no disponen de página web, el promedio del porcentaje de indicadores validados en las 50 entidades restantes fue el $16,8 \%$.

- La entidad que obtuvo la mejor puntuación (la número 14 en orden de cantidades recibidas de mayor a menor) alcanzó el $37,8 \%$.

Con unos resultados insuficientes, las diferencias entre tipologías de entidades u otras características son irrelevantes.

Por tanto, considerando que ninguna entidad llegó ni al $40 \%$ de indicadores validados y que incluso 5 no tienen web, los resultados demuestran que las entidades no están aplicando las obligaciones que les impone la Ley, a pesar de que todas estas reciben cantidades muy elevadas de las administraciones públicas.

\section{Conclusiones}

La transparencia es un valor ineludible y una necesidad para las entidades no lucrativas. No solo resulta fundamental para aumentar la confianza de sus diferentes públicos, para justificar el uso de los fondos públicos recibidos y para cumplir con la Ley, también mejora su eficiencia, previene malas prácticas, mejora la democracia interna, es útil para obtener recursos, aumentar el intercambio con otros entes y aprender del funcionamiento de otras organizaciones similares.

Pero estas no solo no lo están incorporando apropiadamente, tampoco están aplicando la legislación que les obliga a informar y a hacerlo de forma adecuada en beneficio de la ciudadanía en general y de las propias organizaciones. Las entidades no son conscientes de los beneficios que les aporta aplicar el valor de la transparencia, aunque también es cierto que la aprobación de las aún recientes leyes no ha ido acompañada de un esfuerzo 
Transparencia de las entidades no lucrativas: criterios, metodología...

suficiente de sensibilización, información y formación por parte de las administraciones responsables.

Sin una conciencia clara sobre la importancia de aplicar principios de comunicación transparentes, y sin la concurrencia de un régimen sancionador para quienes no cumplen con las obligaciones legales, las rutinas de trabajo en este campo se mantienen e impiden el paso a nuevas prácticas de innovación y mejora que, bien es cierto, requieren un esfuerzo inicial para su implantación y consolidación, basado en una reflexión interna y en acuerdos de todas las partes implicadas: órganos de gobierno, equipos técnicos, socios, colaboradores, voluntarios, etc.

También es necesario contar con recursos suficientes, de los que no se dispone en todos los casos. Si bien las grandes organizaciones deben afrontar sin dificultades este proceso, al contar con profesionales con formación y funciones específicas para las diferentes áreas de trabajo, en muchas otras se cuenta con personal multifunción o incluso con personal voluntario para realizar tareas que han de impulsar la acción, como es el caso de la comunicación. En estas circunstancias, resulta especialmente necesario facilitar a las organizaciones las herramientas que les permitan realizar este trabajo y consolidar una actitud transparente en la organización, instalada sin retorno. Esas herramientas han de facilitar el cumplimiento sin provocar efectos contrarios a los deseados, es decir, sin paralizar el cumplimiento con la misión de la entidad, función que le da sentido.

En esta investigación hemos abordado la transparencia considerando que aporta a las entidades beneficios en tres campos de cuestiones esenciales: conocimiento, democracia y desarrollo. La incorporación de estas prácticas no tiene repercusión tan solo en la entidad, individualmente, ha de suponer un crecimiento y fortalecimiento del sector en su conjunto. Un sector que por su naturaleza y propósitos debe liderar la implantación de valores y de una nueva cultura social fundamentada en la transparencia, que debe extenderse al conjunto de la sociedad. 
Transparencia de las entidades no lucrativas: criterios, metodología...

Partiendo de esta perspectiva, hemos elaborado una metodología, que contiene elementos cuantitativos (información disponible) y cualitativos (aplicando criterios de calidad como el acceso a la información o su inteligibilidad). Permite evaluar la transparencia de las entidades sin ánimo de lucro y también puede ser utilizada por parte de las entidades para obtener un diagnóstico de su situación y, a partir de este, conocer y aplicar un procedimiento de mejora. Se trata, por tanto, de un instrumento de investigación, pero conceptualizado para ser también utilizado por los responsables de las propias organizaciones para mejorar sus prácticas. Con esta finalidad, lo hemos adaptado para poder utilizado a través de plataforma web TransparEnt, confiriendo para ello el procedimiento de mayor flexibilidad y de nuevas características funcionales.

Esta metodología ya ha sido aplicada en el ámbito académico. Por una parte, por el propio equipo que la ha desarrollado, pero también ha sido inmediatamente utilizada por otros investigadores (FRANCÀS GULLÉN; GINESTA PORTET, 2016), lo que prueba la utilidad y solidez de la propuesta.

También la están utilizando las entidades para hacer autoevaluación y para mejorar su comunicación, por lo que podemos validar igualmente el efecto esperado en el sector al que dirige. Desde la presentación de la plataforma (07/04/16) hasta finales de ese mismo año, se había registrado en ella y la estaban utilizando 213 entidades. El seguimiento de la utilización de esta herramienta de intervención nos permite ver cuáles son las organizaciones interesadas. Aunque las hay de diferentes perfiles, el dominante es el de una entidad del sector social que cuenta con un presupuesto de más de 100.000€ anuales, es decir, una entidad obligada a cumplir con la legislación sobre transparencia, con recursos elevados y con una actividad que presenta valores arraigados.

A pesar de esto, hemos comprobado que el nivel de cumplimiento es bajo, tanto por los datos que se desprenden de la misma utilización de la plataforma TransparEnt como de la evaluación realizada a una muestra de entidades que reciben subvenciones o ayudas económicas de las administraciones públicas superiores a 
Transparencia de las entidades no lucrativas: criterios, metodología...

Pedro Molina Rodríguez-Navas • Vanessa Rodríguez-Breijo

$200.000 €$ anuales. De las 55 entidades analizadas, 5 ni siquiera tenían página web. El resto obtuvo una nota media de $16,8 \%$ y la que tuvo una mejor puntuación llegó a un 37,8\%. Estos resultados son similares a los arrojados por investigaciones anteriores (GÁLVEZ RODRÍGUEZ; CABA PÉREZ; LÓPEZ GODOY, 2009, 2012) y con los obtenidos por el Observatori del Tercer Sector (VALLS, 2010). Pero cabía esperar que en este momento de presión social favorable a la transparencia y de responsabilidades legales la tendencia hubiera cambiado de forma clara, fenómeno que no ha sucedido.

Resulta esencial, por tanto, incrementar y profundizar en la sensibilización y formación de los profesionales y responsables de las entidades, poner a su disposición herramientas que faciliten su trabajo y modelos de buenas prácticas. El cambio debe operarse, necesariamente, en la cultura organizacional. De ahí la importancia del asesoramiento y de acciones formativas que se lleven a cabo dentro de esas organizaciones, tal como está contemplado en el proyecto TransparEnt. Con esta finalidad continuamos trabajando y colaborando con las entidades y con las administraciones públicas responsables de la ejecución de la Ley de Transparencia y de la concesión de recursos económicos a las entidades.

\title{
Financiación
}

Esta investigación ha contado con financiación de la Direcció General d'Acció Cívica i Comunitaria de la Generalitat de Cataluña, de la Secretaria de Transparència i Govern Obert de la Generalitat de Cataluña y del Ayuntamiento de Barcelona.

\section{Referencias}

\author{
ARREDONDO TRAPERO, F. G.; GARZA GARCÍA, J. DE LA; VÁZQUEZ \\ PARRA, J. C. TRANSPARENCIA EN LAS ORGANIZACIONES, UNA APROXIMACIÓN DESDE \\ la perspectiva de los colaboradores. Estudios Gerenciales, Cali, v. 30, P. \\ 408-418, 2014.
}


Transparencia de las entidades no lucrativas: criterios, metodología...

Pedro Molina Rodríguez-Navas • Vanessa Rodríguez-Breijo

BAAMONDE-SILVA, X.M.; PENA-RODRÍGUEZ, A.; MARTÍNEZ-ROLÁN, X. LA GESTIÓN DE LA COMUNICACIÓN DIGITAL COMO HERRAMIENTA DE TRANSPARENCIA y ReNDición de Cuentas en la ONGDs. Revista de la Asociación Española de Investigación de la Comunicación, Santiago de Compostela, v. 2, N. 4, P. 27-33, 2015.

BERTOT, J. C.; JAEGER, P.T.; GRIMES, J. M. USING ICTS TO CREATE A CULTURE OF TRANSPARENCY: E-GOVERNMENT AND SOCIAL MEDIA AS OPENNESS AND ANTI-CORRUPTION TOOLS FOR SOCIETIES. GOVERNMENT INFORMATION QUARTERLY, LoNDON, v. 27, N. 3, P. 264-271, 2010.

\section{BLANES CLIMENT, M. A. LA tRANSPARENCIA INFORMATIVA DE LAS}

Administraciones públicas. El Derecho de las Personas a Saber y la

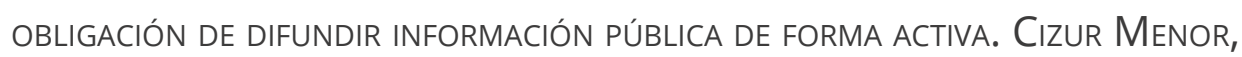
Navarra: Aranzadi Thomson Reuters, 2014.

BORGE BRAVO, R. NueVAS teCnOlogías Y REgENERACIÓN DE LA DEMOCRACIA. En: COTINO HUESO, L. (CoOrd.). Democracia, participación y voto a través de las nuevas tecnologías. Granada: Comares, 2007.

BRANDEIS, L. D. Other People's money and how the bankers use it. NeW York: Frederick A. Stokes Company, 1914.

CALVO, E. COMUNiCACIÓN POLÍTICA 2.0 y BUen GOBieRno. EN: RÖMER PIERETTI, M. (Coord.). Miradas a las pantallas en el bolsillo. Madrid: Universidad Camilo José Cela, 2013.

COMISIÓN EUROPEA. La Gobernanza EUROPEA - un LIBRo blanco. En:

Diario Oficial de las Comunidades Europeas. 428 final, DOCE C-287, DE 12 DE NOVIEMBRE DE 2001. DISPONIBLE EN: HTTP://EUR-LEX.EUROPA.EU/ LEGAL-CONTENT/ES/TXT/PDF/?URI=CELEX:52001DC0428\&RID=1. Consultado el: 01 oct. 2017.

COORDINADORA DE ONG PARA EL DESARROLLO - ESPAÑA. INDICADORES DE TRANSPARENCIA Y BUEN GOBIERNo (VERSIÓN ONGD). MADRID: Publicaciones Coordinadora, 2012. Disponible en: htTP://Webtransparencia. 
Transparencia de las entidades no lucrativas: criterios, metodología...

Pedro Molina Rodríguez-Navas • Vanessa Rodríguez-Breijo

COORDINADORAONGD.ORG/WP-CONTENT/UPLOADS/HERRAMIENTA_TRANSPARENCIA_Y_ Buen_Gobierno_Rev_Marzo_20121.pdf. Consultado el: 10 ост. 2017.

CRESPO RODRÍGUEZ, M.; ZAFRA JIMÉNEZ, A. Transparencia y buen gobierno. Su regulación en España. Madrid: La Ley, 2005.

EOM, S.-J. Improving GOVERnMENTAL TRANSPARENCY IN KoreA: TOWARD institutionalized and ICT-enabled transparency. The Korean Journal of Policy Studies, Seoul, v. 29, N. 1, P. 69-100, 2014.

EUROPEAN COMISSION. Europe 2020. A strategy for SMART, sustainable AND INCLUSive growth. Brussels: European Comission, 2010. DISPONIBLE EN: HTTP://EUR-LEX.EUROPA.EU/LEGAL-CONTENT/ES/TXT/PDF/?URI=CE LEX:52010DC2020\&from=en. Consultado el 01 oct. 2017.

FERNÁNDEZ, R.; OSPINA, R. I. Manual de autoevaluación sobre las PRÁcticas de transparencia y Rendición de Cuentas. Medellín, Colombia: Iniciativa Regional de la sociedad Civil/Rendir Cuentas, 2012. Disponible EN: HTTP://RENDIRCUENTAS.ORG/APLICACIONRENDIRCUENTAS/INDEX.HTML.

Consultado el 10 oct. 2017.

FERNÁNDEZ TORRES, M. J. GESTIÓN DE LA COMUNICACIÓN EN EL SECTOR NO lucrativo español. Revista de Comunicación de la SEECI, Madrid, v. XVII, N. 30, P. 94-105, 2013.

FRANCÀS GULLÉN, B.; GINESTA PORTET, X. LA TRANSPARENCIA EN las entidades del Tercer Sector en Cataluña. Un análisis a partir de la INFORMACIÓN PUBLICADA EN SUS WEBS CORPORATIVAS. EN: LARRONDO URETA, A.; MESO AYERDI, K.; PEÑA FERNÁNDEZ, S. (EDS.). EL IMPACTO dE LAS AUDIENCIAS EN LOS PERFILES PROFESIONALES Y LOS CONTENIDOS, VIII CONGRESO Internacional de Ciberperiodismo. BilbaO: Servicio Editorial Universidad del País VASCO, 2016.

GÁLVEZ RODRÍGUEZ, M. M.; CABA PÉREZ, M. C.; LÓPEZ GODOY, M. La tRansparencia on-line de las ONG españolas. Revista Española del Tercer Sector, Madrid, v. 13, P. 63-86, 2009. 
Transparencia de las entidades no lucrativas: criterios, metodología...

Pedro Molina Rodríguez-Navas • Vanessa Rodríguez-Breijo

GÁLVEZ RODRÍGUEZ, M. M.; CABA PÉREZ, M. C.; LÓPEZ GODOY, M. RESPONSABILIDAD SOCIAL Y TRANSPARENCIA ON-LINE DE LAS ONG: ANÁLISIS del caso español. CiRIEC-España, Revista de Economía Pública, Social y Cooperativa, Valencia, v. 74, P. 207-238, 2012.

GARCÍA COSTA, F. M. PARTICIPACIÓN Y DEMOCRACIA ELECTRÓNICAS EN EL Estado representativo. En: COtino hUeSO, L. (Coord.). Democracia, PARTICIPACIÓN Y VOTO A tRAVÉS de LAS NUEVAS TeCnOlogías. GRANADA: Comares, 2007.

GAVENTA, J.; MCGEE, R. The IMPACT OF TRANSPARENCY AND ACCOUNTABILITY initiatives. Development Policy Review, London, v. 31, N. 1, P. s3-s28, 2013.

\section{GENERALITAT DE CATALUNYA. Guía de Tranparència Per a les} ASSOCIACIONS I FUNDACIONS PERCEPTORES DE SUBVENCIONS I AJUTS PÚBLICS. Barcelona: Generaltitat de Catalunya, Comissionst per a la Transparència I l'Accés a la Informació Pública, 2015. Disponible en: htTp://Justicia. GENCAT.CAT/CA/AMBITS/DRET_I_ENTITATS_JURIDIQUES/TRANSPARENCIA_ENTITATS/. Consultado el 01 oct. 2017.

GUTTERMAN, E. The Legitimacy of transnational NGOs: LESSONS FROM the experience of Transparency International in Germany and France. Review of International Studies, ShefField, v. 40, P. 391-418, 2013.

HERRANZ DE LA CASA, J. M. LA GESTIÓN DE LA COMUNICACIÓN COMO ELEMENTO GENERADOR DE TRANSPARENCIA EN LAS ORGANIZACIONES NO LUCRATIVAS. CIRIECEspaña, Revista de Economía Pública, Social y Cooperativa, Valencia, v. 57, P. 5-31, 2007.

KOSTADINOVA, P. IMPROVING THE TRANSPARENCY AND ACCOUNTABILITY OF EU InStitUTIONS: THE IMPACT OF THE OfFICE OF THE EUROPEAN OMBUdSMAN. JouRnal of Common Market Studies, Kent, v. 53, N. 5, p. 1077-1093, 2015.

LAPORTE, T. M.; DEMCHAK, C. C.; DEJONG, M. Democracy and bureaucracy in the age of the Web. Administration \& Society, Virginia, v. 34, N. 4, P. 411-446, 2002. 
Transparencia de las entidades no lucrativas: criterios, metodología...

Pedro Molina Rodríguez-Navas • Vanessa Rodríguez-Breijo

MOLINA RODRÍGUEZ-NAVAS, P.; SIMELIO SOLÀ, N.; CORCOY RIUS, M. Metodologías de eVAluación de LA tRanSPAREnCia: PROCEDIMIENTOS Y PROblemas. Revista Latina de Comunicación Social, La Laguna (Tenerife), N. 72, P. 818-831, 2017.

MORENO SARDÀ, A. ET AL. INFOPARTICIP@: PERIODISMO PARA LA PARTICIPACIÓN Ciudadana en el Control democrático. CRiterios, metodologías Y

herRamientas. Estudios sobre el mensaje Periodístico, Madrid, v. 19, N. 2, P. 783-803, 2013.

ROBERTS, A. Blacked out: GOVERNMENT SECRECY IN THE INFORMATION AGE. New York: Cambridge University Press, 2006.

ROCHA VALENCIA, L. A.; QUEIRUGA, D.; GONZÁLEZ BENITO, J. RELATIONSHIP BETWEEN TRANSPARENCY AND EFFICIENCY IN THE ALLOCATION OF FUNDS IN NONGOVERNMENTAL DEVELOPMENT ORGANIZATIONS. VoluntAS: INTERNATIONAL Journal of Voluntary and Nonprofit Organizations, Vienna, v. 26, N. 6 , P. 2517-2535, 2015.

SCHAUER, F. Transparency in three Dimensions. University OF Illinois LaW Review, ILLINOIS, V. 4, P. 1339-1358, 2011.

United nations. 55/2. United Nations Millennium Declaration. A/ ReS/55/2. New York: United Nations, General Assembly, 8 de Setiembre de 2000. DisPONIBLE EN: HTTP://WWW.UN.ORG/MILLENNIUM/DECLARATION/ARES552E. htm. Consultado el 01 oct. 2017.

VALLS, N. LA TRANSPARÈNCIA I LA RENDICIÓ DE COMPTES AL TERCER SECTOR.

Reflexió I autodiagnòstic. Barcelona: Observatori del Tercer Sector, 2010. 